\title{
Lifeworks
}

\section{Dancing with research}

A t first glance, the art of dance has found an unlikely partner in health research. But upon reflection, one can see at least 2 points of commonality: both involve interpretation - of emotion and illness, respectively - through the physical body and both involve forms of communication. These commonalities are $\infty$ the starting point for a relatively new art variation: dance, accompanied by music, that provides a visual and aural vocabulary through which the subjective experiences of mental health and other patients can be demonstrated and research results disseminated.
There are several advantages to this unusual approach, including the fact that while research results are predominantly communicated through academic journals and conferences, dance is accessible to everyone. And perhaps more important is the fact that the subjective experiences of clients are not typically included in the reporting of research results. This is particularly pertinent in the context of mental health research.

Readers may be familiar with the use of dance as an adjunct to psychotherapy to facilitate the development of social skills and heighten the sense of well-being in mental health clients. In addition, mental illness has been interpreted through dance by various modern dance troupes. Dance is also used in some African communities to communicate health care issues surrounding HIV/AIDS.

Social issues have also been examined through dance. For example, in Canada, choreographer Judith Marcuse used a shopping mall as a backdrop for 2 dances: one aimed at helping young people to understand the effects surrounding teen suicide, the second examining the presence of violence and its effects on the lives of youth.

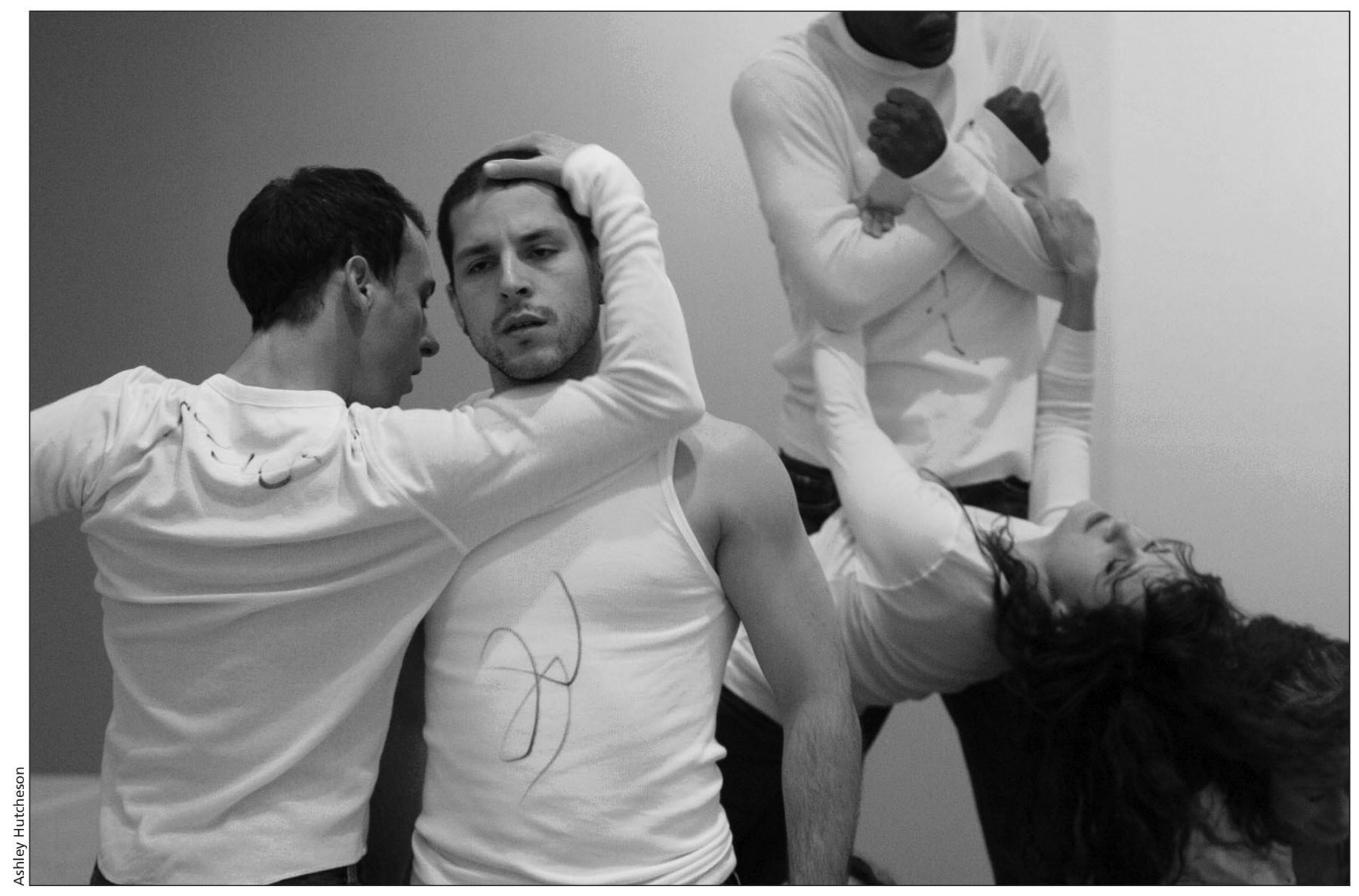

Dancers in Hearing Voices add the emotional, subjective experience to the dissemination of research about the "pathways" to treatment for young people (aged 14 to 24) suffering with psychosis. 
In addition, the art of dance has proved useful in disseminating scientific ideas and values. For example, in the United States, Liz Lerman's Dance Exchange troupe is exploring the repercussions of knowledge of the human genome through its dance, Ferocious Genome, which centres on the values behind perfection and the effects of ancestry. Thirty-four genetic scientists and researchers collaborated on the dance project with Dr. Francis S. Collins facilitating a postdance discussion.

More recently, however, the movements of dance are being used to communicate both research results and the client's subjective experiences, so that the "objective" and the "subjective" act as metaphorical partners to each other.

One such dance performance, presented at the Convergence of Art and Science: Global Health Perspectives conference, June 4-6, 2008, in Toronto, Ontario, was the result of a collaboration between Katherine Boydell PhD, a sociologist and senior scientist at the Child Health Evaluative Sciences Research Institute at The Hospital for Sick Children in Toronto, and choreographer Siona Jackson.

Boydell said she chose dance as the medium as she believes in its "power to communicate." The dance, entitled Hearing Voices, was based on her research examining the "pathways" that young people (aged 14 to 24), suffering from a first episode of psychosis, took to receive mental health care, and Boydell's concerns regarding whether research results affect service providers.

Boydell conducted 60 interviews with young people, as well as their family members and individuals whom the young people viewed as either helpful or not helpful in directing them to professional help. She found that young people's search for mental health care is "complex and not a linear pathway, [but more] like a topographical map" and that it typically took 1 to 2 years before the young person sought help.

In addition, she found that the symptoms of young people are often dismissed as "teenage angst" and that the young people themselves often tried to deal with their symptoms, such as paranoia and hearing voices, by avoiding people.

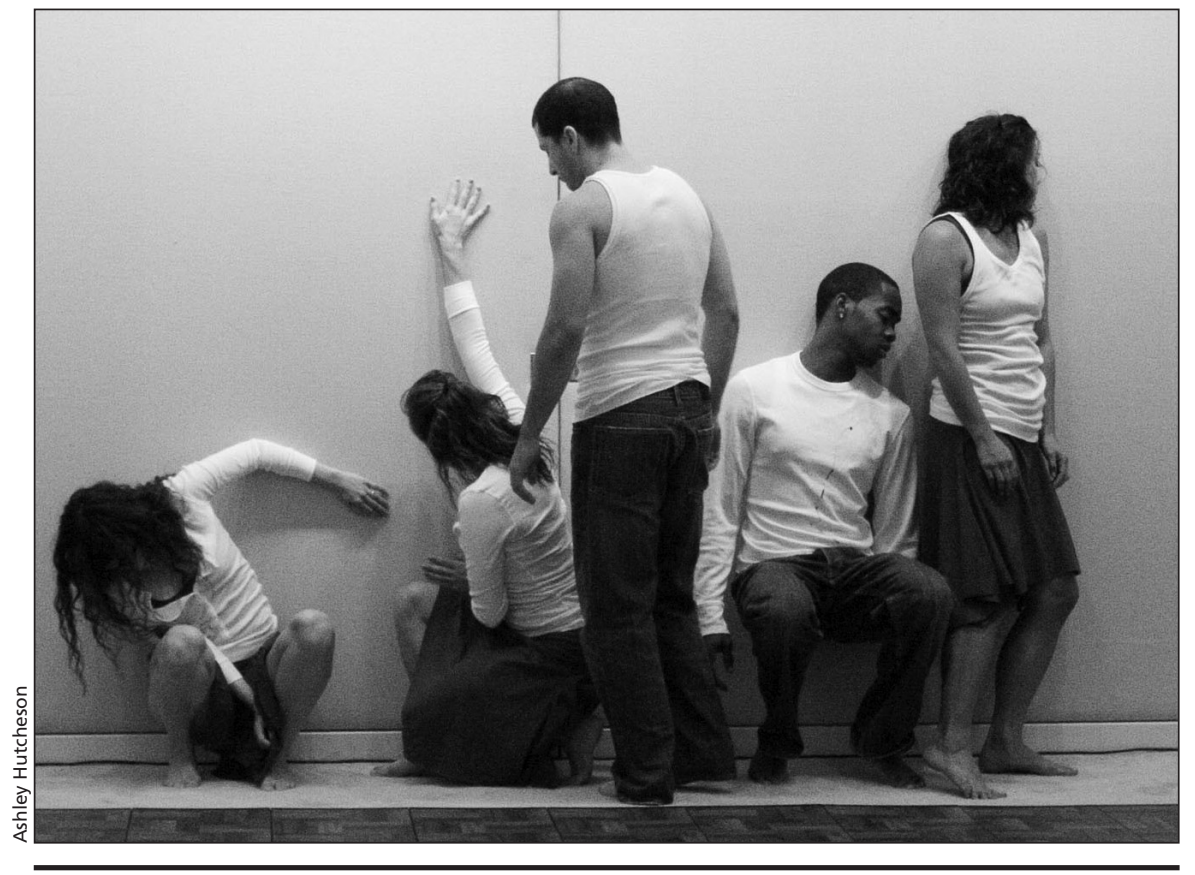

These dancers in Hearing Voices communicate the feeling of isolation that was articulated by many of the young people who participated in a research survey.

The dance communicating these results was highly provocative, as dancers moved with jarring, angular movements, and sometimes collided or moved violently. The accompanying voice-over was based on transcripts of interviews with young people who were suffering first-episode psychosis. Visually and aurally the dancers' performance communicated the sense of alienation and disconnection experienced by an individual with psychosis. Their movement embodied the emotional roller coaster of psychosis that research, on paper, cannot communicate as fluently to a nonprofessional audience.

Performances of Hearing Voices have also been staged during grand rounds at The Hospital for Sick Children in Toronto and a symposium for qualitative inquiry on early psychosis, as well as for a group of high-risk secondary students from across Ontario. Boydell says that she would now like to see the danced performed at rural, urban and inner-city high schools.

After each performance, audience members were invited to provide feedback. One viewer wrote that the performance would be beneficial to children exposed to war, such as those in Bosnia. Another wrote "I was skeptical and I was transformed." Still another commented: "The dance could communicate information about psychosis that was not stigmatizing."

Dr. John S. Strauss, professor emeritus, psychiatry at Yale University, New Haven, Connecticut, wrote: "Getting a clinician to understand the experience of psychosis requires a variety of ways of helping him or her 'be there.' The dance performance is for some people a powerful way to do that."

\section{J. Lynn Fraser BA(d) MES}

Textile artist and writer

Toronto, Ont.

\section{FURTHER READING}

- Bagley C, Cancienne MB. Educational research and inter-textual forms of (re)presentation: the case for dancing the data. In: Bagley C, Cancienne MB, editors. Dancing the data. New York: Peter Lang; 2002. p. 3-19.

- Cancienne MB. From research analysis to performance: the choreographic process. In: Knowles JG, Cole AL, editors. Handbook of the arts in qualitative research: perspectives, methodologies, examples, and issues. Thousand Oaks (CA): Sage Publications; 2008. p. 405

- Finley S. Arts-based inquiry: performing revolutionary pedagogy. In: Denzin NK, Lincoln YS, editors. The Sage handbook of qualitative research. 3rd ed. Thousand Oaks (CA): Sage Publications; 2005. p. 681-94.

- $\quad$ Norris JR. Meaning through form: alternative modes of knowledge representation. In: Morse JM, editor. Completing a qualitative project: details and dialogue. Thousand Oaks (CA): Sage Publications; 1997. p. 87-115. 